\title{
Post-Activation Potentiation Increases Recruitment of Fast Twitch Fibers: A Potential Practical Application in Runners
}

\author{
by \\ Marco Gervasi ${ }^{1}$, Anna Rita Calavalle ${ }^{1}$, Stefano Amatori ${ }^{1}$,Eugenio Grassi ${ }^{1}$, Piero \\ Benelli ${ }^{1}$, Piero Sestili ${ }^{1}$, Davide Sisti ${ }^{1}$
}

To determine the relationship between fatigue and post-activation potentiation, we examined the effects of submaximal continuous running on neuromuscular function tests, as well as on the squat jump and counter movement jump in endurance athletes. The height of the squat jump and counter movement jump and the estimate of the fast twitch fiber recruiting capabilities were assessed in seven male middle distance runners before and after 40 min of continuous running at an intensity corresponding to the individual lactate threshold. The same test was then repeated after three weeks of specific aerobic training. Since the three variables were strongly correlated, only the estimate of the fast twitch fiber was considered for the results. The subjects showed a significant improvement in the fast twitch fiber recruitment percentage after the $40 \mathrm{~min}$ run. Our data show that submaximal physical exercise determined a change in fast twitch muscle fiber recruitment patterns observed when subjects performed vertical jumps; however, this recruitment capacity was proportional to the subjects' individual fast twitch muscle fiber profiles measured before the 40 min run. The results of the jump tests did not change significantly after the three-week training period. These results suggest that pre-fatigue methods, through sub-maximal exercises, could be used to take advantage of explosive capacity in middle-distance runners.

Key words: squat jump, middle distance running, lactate threshold, fibers, recruitment.

\section{Introduction}

Neuromuscular function tests, such as a squat, counter movement and drop jumps, maximal isometric voluntary contraction (MVIC) and isokinetic maximal concentric contractions (Abernethy et al., 1995; Cometti et al., 2011; Wilson and Murphy, 1996), are important tools for monitoring fatigue in athletes (Raeder et al., 2016; Maszczyk et al., 2016; Sams et al., 2018); however, there is no standardized test to measure muscle fatigue in different sports (Baar and Mcgee, 2008). Several studies have shown a decrease in maximal voluntary contraction (MVC) after long duration physical activities such as marathons or ultramarathons (Giovanelli et al., 2016; Millet et al., 2002; Millet and Lepers, 2004). Moreover, it is known that maximal strength is produced by the neuromuscular recruitment of both fast and slow twitch fibers (Bosco and Komi, 1979); therefore, measuring the MVC peak does not allow to discriminate among the relative contributions of different types of fibers. Bosco and Komi demonstrated that subjects with a high percentage of fast twitch fibers (FTF) in their lower limbs muscles achieved better results in vertical jump tests: the squat jump (SJ) and counter movement jump (CMJ) (Bosco and Komi, 1979; Bosco et al., 1983). Hence, the intervention of phasic units in the maximal execution of this ballistic exercise is dominant over tonic units. Moreover, according to Bosco (1999), the SJ and CMJ, performed at the maximum power output, allow to obtain an indirect estimate of the percentage of FTF recruited, with an error of less than $5 \%$.

The effect of fatigue induced by running exercises on jumping ability is not well

1 - Department of Biomolecular Sciences, University of Urbino Carlo Bo, Urbino, Italy. 
understood. While some authors have reported an acute enhancement of jumping ability (Boullosa et al., 2011; García-Pinillos et al., 2015; LatorreRomán et al., 2014; Vuorimaa et al., 2006) after running protocols in runners, others (Boullosa and Tuimil, 2009) have not found post-activation potentiation (PAP) effects in non-runners after a similar stimulus.

The term of PAP refers to the significant enhancement of muscular twitch force after voluntary contractile activity (Mettler and Griffin, 2012), but the mechanism responsible for this muscle potentiation has not been fully elucidated (Wilson et al., 2013; Gołaś et al., 2016). There are several suggested mechanisms behind PAP, including the recruitment of higher order motor units, an increase in the pennation angle, and the phosphorylation of myosin regulatory light chains (Healy and Comyns, 2017; Tillin and Bishop, 2009). PAP also appears to occur at the spinal level, through increased synaptic efficacy between Ia afferent terminals and $\alpha$-motoneurons of the homonymous muscle (Hodgson et al., 2005). Regardless of the mechanism involved, the aim of incorporating PAP protocols into an athlete's training program is to elicit an acute enhancement in performance.

There is also a lack of information on the mechanism responsible for the PAP phenomenon induced by running exercises in endurance runners. Among the studies that found PAP after submaximal exercise, Vuorimaa et al. (2006) showed that $40 \mathrm{~min}$ of running at a speed corresponding to $80 \% \mathrm{VO}_{2 \max }$ produced significant improvement in power performance of elite longdistance runners. However, the authors gauged intensity by maximum oxygen uptake, without taking into account the individual lactate threshold of the subjects. In this regard, a study performed by Meyer et al. (1999) has shown that, due to the large variability of individual values of $\mathrm{VO}_{2 \max }$ and $\mathrm{HR}$ at the lactate threshold, for the control of elite athletes or for research purposes, it is important to calculate intensity using a percentage of the lactate threshold and not $\mathrm{VO}_{2 \max }$ or HRmax.

The first aim of our study was to evaluate vertical jumping ability and to estimate the percentage of FTF involved in the jump using the Bosco's test (Bosco, 1999), before and after $40 \mathrm{~min}$ of running on a treadmill at an intensity equal to the speed of the individual lactate threshold, namely LT2 $(4.0 \mathrm{mmol} / \mathrm{l})$. The second aim was to test whether this effect changed after 3 weeks of specific training.

\section{Methods}

\section{Experimental Approach to the Problem}

This study tested the hypothesis that a standardized submaximal run at an intensity equal to the speed of LT2, able to affect a selective glycogen depletion in the STF (Seiler and Kjerland, 2006), could improve the FTF recruitment ability under pre-fatigued muscle conditions.

Subjects

Seven male athletes were enrolled, age $26.6 \pm 6$ years; body mass $66.7 \pm 7 \mathrm{~kg}$; body height $177.8 \pm 8 \mathrm{~cm}$, with training experience in the extended middle-distance events of $4 \pm 1$ years. The subjects provided written informed consent in accordance with the ethics committee of the University of Urbino. The study was approved by the Department of Biomolecular Science of the Urbino University and was conducted in agreement with the Declaration of Helsinki (1975).

\section{Procedures}

Each athlete was tested to determine his LT2 and individual training zones. Training intensity zones for each athlete were based on the following lactate concentration values: zone 1 (< $\left.\mathrm{LT}_{1}\right),<2.0 \mathrm{mmol} / \mathrm{L}$, zone $2\left(>\mathrm{LT}_{1}<\mathrm{LT}_{2}\right),>2.0$ and $<4.0 \mathrm{mmol} / \mathrm{l}$, zone $3\left(>\mathrm{LT}_{2}\right),>4.0 \mathrm{mmol} / \mathrm{l}$, as proposed by Seiler and Kjerland (2006).

The protocol to determine the LT2 consisted of a minimum of 5 and a maximum of 9 incremental $4 \mathrm{~min}$ stages. The intensity of the first step was calculated according to the individual athletic level, and the speed of the treadmill was increased by $1 \mathrm{~km} / \mathrm{h}$ every $4 \mathrm{~min}$ as indicated in the guidelines (Winter, 2006). Lactate concentration was measured by microwithdrawals from the fingertip of the forefinger with a Lactate Pro lactate meter (ARKRAY, Kyoto, Japan) (Baldari et al., 2009; Tanner et al., 2010) before the test and within the $30 \mathrm{~s}$ of the end of each stage. The running speed at LT2 $(4.0 \mathrm{mmol} / \mathrm{l})$ was determined using a validated algorithm by Bentley et al. (2007) and implemented using specifically designed software by Newell et al. (2007). Approximately $20 \mathrm{~min}$ after the lactic threshold tests, the athletes were shown the 
proper execution of the SJ and CMJ. The athletes returned to the lab for the experimental phase of the study 48 hours after the initial meeting.

The subjects performed five SJs and CMJs, before $\left(\mathrm{t}_{0}\right)$ and $3 \mathrm{~min}$ after $\left(\mathrm{t}_{1}\right)$ the $40 \mathrm{~min}$ run on the treadmill at LT2 speed. Performance was measured using an Ergojump contact platform (Ergojump, Psion XP, MA.GI.CA., Rome, Italy) as described by Bosco et al. (1983). This Jump-mat system provides trustworthy measurements for monitoring changes in jump height (Pueo et al., 2016). Before performing the $40 \mathrm{~min}$ run at LT2, the athletes completed a $10 \mathrm{~min}$ warm up at a speed of $10 \mathrm{~km} / \mathrm{h}$. After 3 weeks of specific training, the SJ and CMJ tests were repeated before ( $\left.\mathrm{t}_{2}\right)$ and after ( $\left.\mathrm{t}_{3}\right)$ a 40 min run at LT2, newly defined using the same protocol as in the first test. Differences in speed at LT2 before and after the training period are shown in Figure 1.

\section{Statistical Analyses}

The measured variables, tested at the beginning of the experiment and after the threeweek training period, were: height of the SJ and CMJ, estimate of FTF percentage recruitment (\%FTF) (Bosco, 1992) using the height of the jump tests, running speed at LT2.

In order to evaluate the strength of the correlation among the SJ, CMJ and \%FTF, regression analysis was performed. Since the variables $\mathrm{SJ}, \mathrm{CMJ}$ and \%FTF were strongly correlated (Figure 2), only the \% FTF variable was considered.

The \%FTF data are provided in a scatter plot: we calculated the linear regression from the values at $t_{0}$ and $t_{1}$, and the same was done for the values at $t_{2}$ and $t_{3}$. In order to highlight the significance of the differences between $t_{0}-t_{1}$, and between $t_{2}-t_{3}$, the statistic of the difference between the slopes and the intercepts of the regression lines was used. The slope of the regression line between $t_{0}$ and $t_{1}$ was then compared to the null hypothesis $(b=1)$ to determine if there was a significant change in the percentage of fast fibers recruited; the same procedure was performed for $t_{2}$ and $t_{3}$. Moreover, the two intercepts and the two slopes between the straight lines of linear regression $t_{0}-t_{1}$ and $t_{2}-t_{3}$ were compared (the first error type was set at $0.05)$. Finally, 2-tailed paired sample t-tests were used to determine differences in running speed at LT2 before and after the three-week training period.

\section{Results}

During the training period ( 3 weeks), each athlete had a total of 21 training sessions. Based on the total time-in-zone method, 73.7\% (566 min) of all training time was spent below the $\mathrm{LT}_{1}$ intensity (zone 1), 9.8\% (75 $\mathrm{min}$ ) of endurance training time was between $\mathrm{LT}_{1}$ and $\mathrm{LT}_{2}$ (zone 2), and $16.5 \%$ (127 $\mathrm{min}$ ) was over $\mathrm{LT}_{2}$ (zone 3). The training program is shown in Table 1 , and training intensity distribution is shown in Figure 3.

After 3 weeks of training, the running speed at LT2 varied significantly: $14.1 \pm 0.7$ pretraining vs $14.6 \pm 0.6$ post-training $(p<0.001)$, which accounted for an increase of $3.9 \%$ (Figure $1)$. We did not find significant differences $(p>$ 0.05 ) for the intercepts and the slopes or when comparing the two lines of linear regression $t_{0}-t_{1}$ and $t_{2}-t_{3}$. This means that after 3 weeks of training pre-post \% FTF recruited did not change (Figure 4). With regard to the intercepts (expected value $\mathrm{a}=0$ ) (indicator of systematic increase), there were no significant differences between before and after $40 \mathrm{~min}$ of running, regardless of the training period ( $t_{0}-t_{1}$ versus $\left.t_{2}-t_{3}\right)$. In contrast, the slopes are significantly different from $b=1$ (in dashed line), between $t_{0}$ and $t_{1}$ there is a proportional increase rate of $29 \%(p=0.043)$ and an increase rate of $14 \%$ between $\mathrm{t}_{2}$ and $\mathrm{t}_{3}(p=$ 0.056). In fact, in the latter case we are at the limit of significance, and this is probably due to the low sample size. It should be emphasized that the bivariate distributions considering to and $t_{1}$ and $t_{2}$ and $t_{3}$ are strictly linear $(r=0.97, r=0.99$, respectively) (Figure 4).

\section{Discussion}

PAP has not been explored thoroughly enough in scientific literature, and the causes of this phenomenon have not yet been clarified. García-Pinillos et al. (2016) investigated whether kinematic data during the $\mathrm{CMJ}$ might explain the PAP phenomenon after an exhausting running test; they also hypothesized that improvements observed in CMJ performance despite high fatigue levels, might be related to the manner in which the athletes jumped (jumping kinematic). However, they concluded that the CMJ kinematics did not explain the PAP phenomenon after an 
exhausting running test in endurance runners and rejected the hypothesis that kinematic variables were determinant of the PAP phenomenon in those subjects. Therefore, the causes of PAP must be further investigated under different experimental conditions.
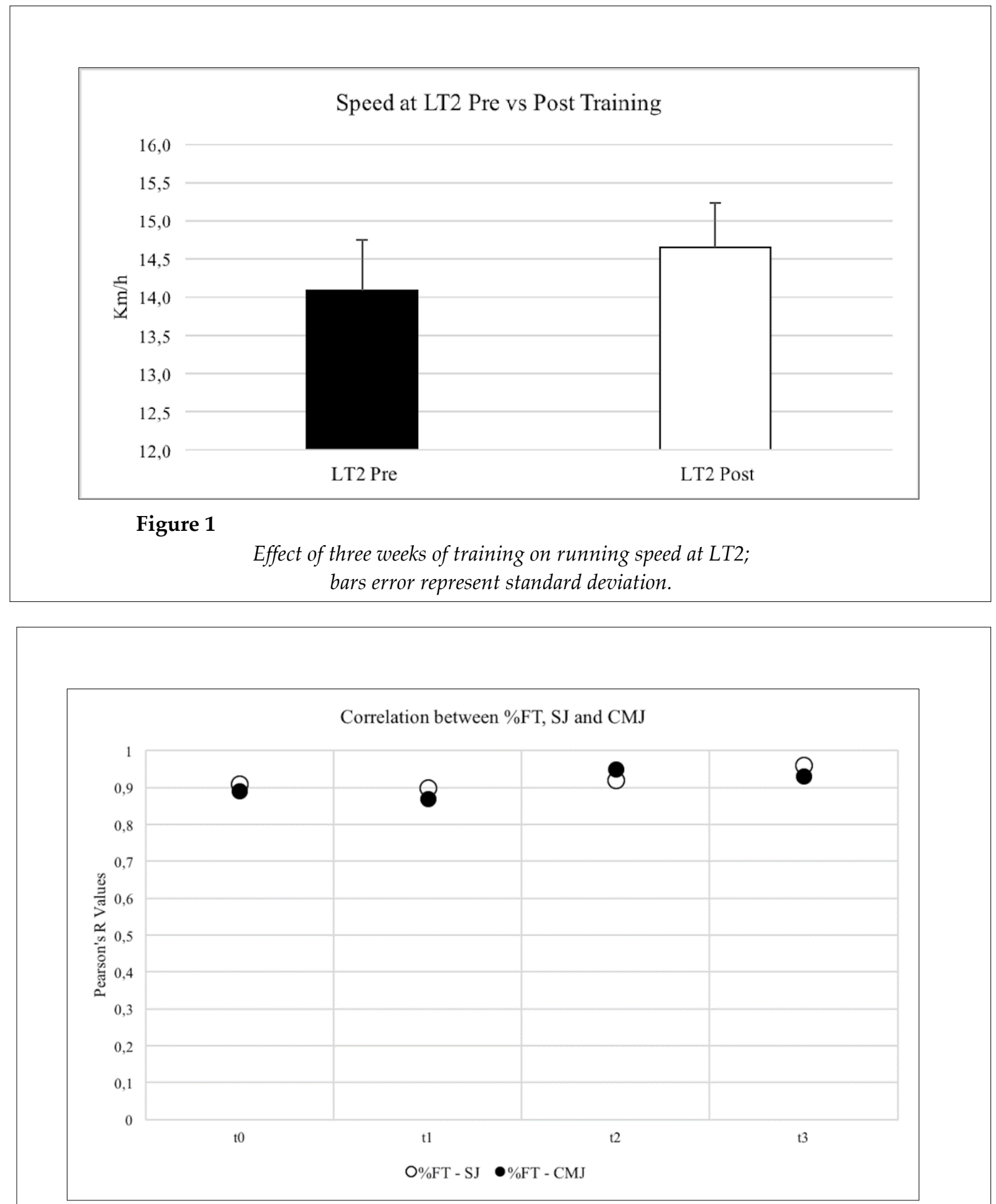

Figure 2

Correlation between SJ, CMJ and \% of FT fibers recruited. 


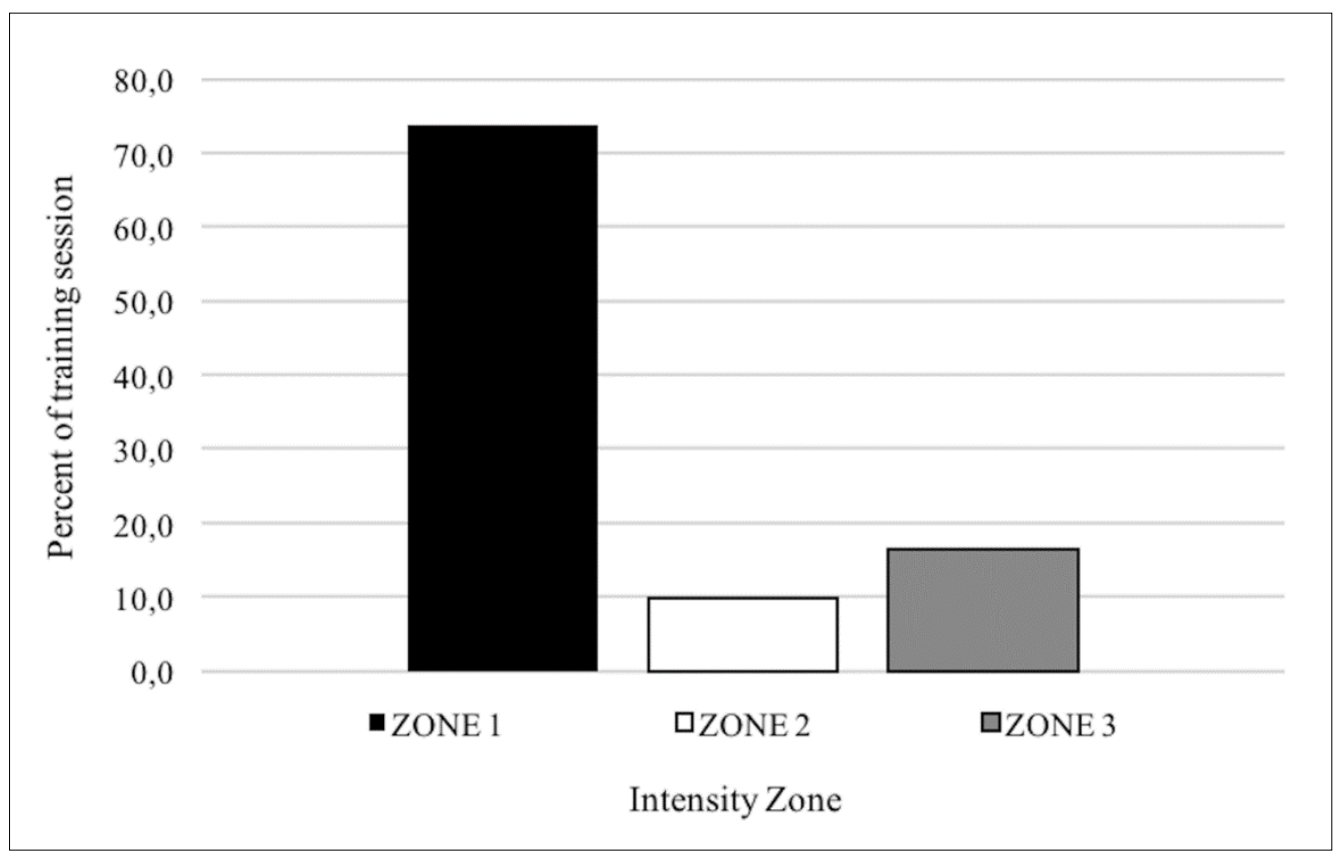

Figure 3

Training intensity distribution in the three zone model during the three-week training period.
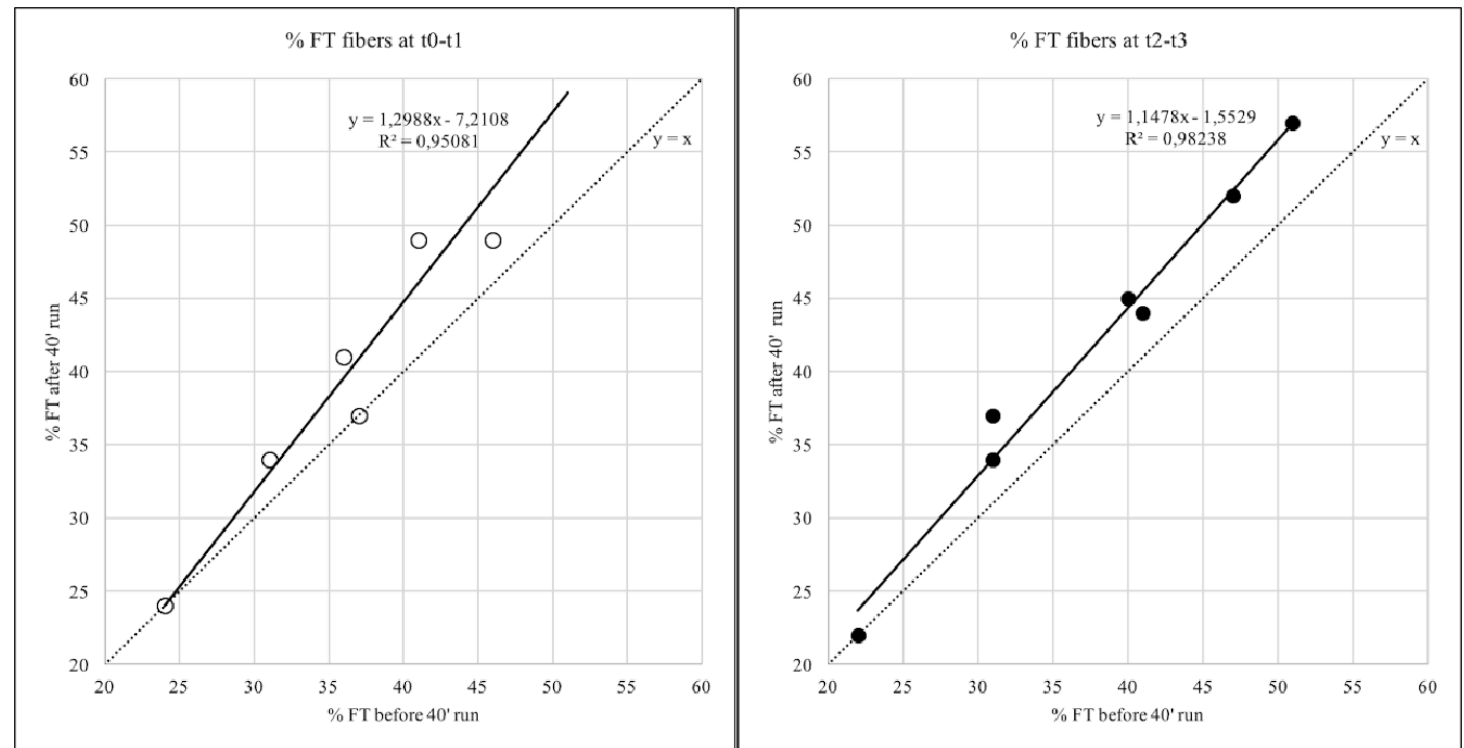

Figure 4

Percentage of FT fibers pre- and post- 40 min run, before and after three weeks of training. 


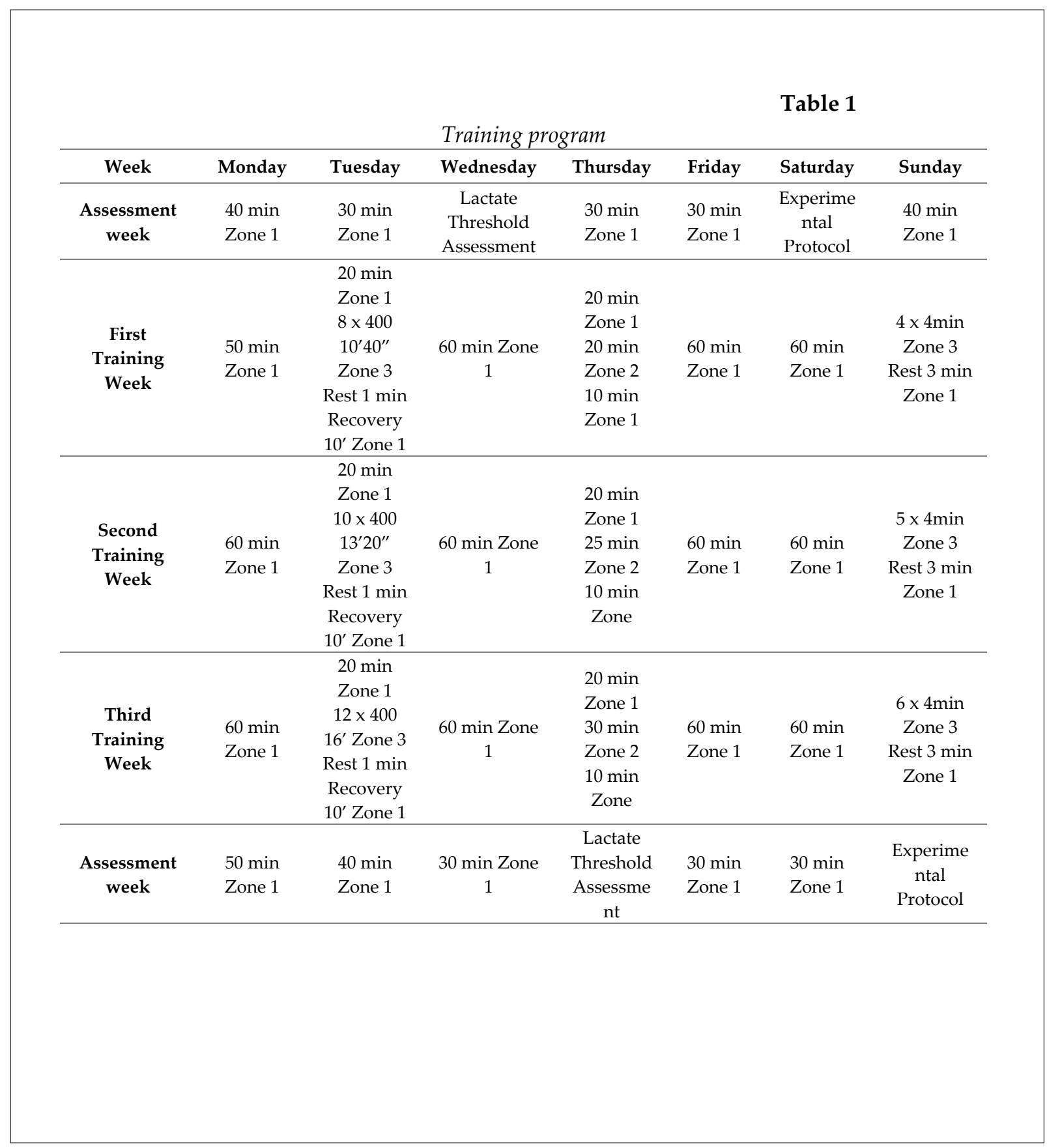

The causes of loss of strength can be divided into central and peripheral causes. With regard to the peripheral causes, the depletion of muscle glycogen is certainly among the most important. It is known that prolonged exercise performed at submaximal intensity causes a selective depletion of muscle glycogen in proportion to the intensity of the exercise (Gollnick et al., 1974). In particular, Gollnick et al. (1974) clearly showed that differential rates of glycogen depletion occurred in human skeletal muscle fibers during exercise with workloads requiring less than $100 \%$ of $\mathrm{VO}_{2 m a x}$ : slow twitch fibers (STF) were the first to lose glycogen, whilst with supramaximal loads (over $100 \% \mathrm{VO}_{2 \max }$ ), the depletion of glycogen occurred first in the FTF. In a more recent study, Krustrup et al. (2004) demonstrated that previous exercise that induced STF glycogen depletion could be used as a model to enhance FTF recruitment during subsequent moderate - intensity exercise. Furthermore, current literature states that there is an increase in the activity of the central nervous system (CNS) proportional to the duration and intensity of the 
endurance exercise (Baar and Mcgee, 2008).

In light of these findings, we hypothesized that a pre-fatigue state obtained by a sub-maximal exercise might yield a selective glycogen depletion of STF, while maintaining high levels of glycogen in FTF. In our study, the tested athletes showed a significant improvement in the estimated recruitment percentage of FTF (Bosco, 1992) during the jump tests following 40 min of running at LT2 speed. After a 3-week training period, despite an increase in running speed at LT2, the results of the jump tests did not change significantly. Hence, we can speculate that the significant increase in the recruitment percentage of FTF observed in our subjects may have been triggered firstly, by an unfavorable energetic condition of STF caused by a selective glycogen depletion following the $40 \mathrm{~min}$ of running at LT2 speed and, secondly, by an increased activity of the CNS resulting in better recruitment of FTF at a high activation threshold. Finally, it should be pointed out that the recruitment of FTF occurs in proportion to the percentage of each subject's FTF. Therefore, subjects with more FTF experience better recruitment of those fibers immediately after the 40-min run, as is apparent from the values reported in the graph (Figure 4). Moreover, our results are confirmed by some studies showing that the most important muscle characteristic affecting PAP is the fiber type; muscles with the shortest twitch contraction times and highest proportion of fast twitch (Type II) fibers show the greatest PAP. The greater PAP in Type II fibers is likely related to their greater capacity for MLC phosphorylation in response to high frequency activation (Hamada et al., 2000).

Despite the limitations of this investigation stemming from the small sample size and the lack of analysis of muscle glycogen measurement, our findings may lay the groundwork for further studies designed to explore and test our hypotheses more thoroughly.

\section{Practical Applications}

Often, during prolonged long-distance races $(5000 \mathrm{~m}, 10000 \mathrm{~m})$, the last lap is run at speeds that require greater recruitment of FTF, and there are many examples of top level athletes who manage to run that final lap in a time that approaches their personal best in the 400 meters. Our study shows that "pre-fatigue" can be used before workouts that require use of FTF (such as explosive force, or even sprinting), in order to improve the final part of a competition or a change of pace. It might therefore be useful, during fractionated training, to include the fastest fractions in the final part of the session (eg. 10000 m. fractionated: $3 \times 2000 \mathrm{~m}+3 \times 1000 \mathrm{~m}+5 \times 200$ $\mathrm{m})$.

\section{References}

Abernethy P, Wilson G, Logan P. Strength and power assessment: issues, controversies and challenges. Sports Med, 1995; 19(6): 401-17

Baar K, Mcgee S. Optimizing training adaptations by manipulating glycogen. European of Sport Science, 2008; 8: 97-106

Baldari C, Bonavolontà V, Emerenziani GP, Gallotta MC, Silva AJ, Guidetti L. Accuracy, reliability, linearity of Accutrend and Lactate Pro versus EBIO plus analyzer. Eur J Appl Physiol, 2009; 107: 105-111

Bentley DJ, Newell J, Bishop D. Incremental exercise test design and analysis: implications for performance diagnostics in endurance athletes. Sports Med, 2007; 37: 575-86

Bosco C, Komi PV. Mechanical Characteristics and Fiber Composition of Human Leg Extensor Muscle. Eur J Appl Physiol, 1979; 41: 275-284

Bosco C, Komi PV, Tihanyi J, Fekete G, Apor P. Mechanical power test and fiber composition of human leg extensor muscles. Eur J Appl Physiol Occup Physiol, 1983; 51(1): 129-35

Bosco C, Luhtanen P, Komi PV. A simple method for measurement of mechanical power in jumping. European Journal of Applied Physiology, 1983; 50: 273-282

Bosco C. Strength assessment with the Bosco's test. Rome: Italian society of sport science; 1999 
Boullosa DA, Tuimil JL. Postactivation potentiation in distance runners after two different field running protocols. Journal of Strength and Conditioning Research, 2009; 23: 1560-1565

Boullosa DA, Tuimil JL, Alegre LM, Iglesias E, Lusquiños F. Concurrent fatigue and potentiation in endurance athletes. Int Jour Sports Physiol Perf, 2011; 6: 82-93

Cometti C, Deley G, Babault N. Effects of Between-Set Interventions on Neuromuscular Function During Isokinetic Maximal Concentric Contractions of the Knee Extensors. J Sports Sci Med, 2011; 10(4): 624629

García-Pinillos F, Soto-Hermoso VM, Latorre-Román PA. Acute effects of extended interval training on countermovement jump and handgrip strength performance in endurance athletes: postactivation potentiation. J Strength Cond Res, 2015; 29: 11-21

Garcia-Pinillos F, Molina-Molina A, Latorre-Roman PA. Impact of an incremental running test on jumping kinematics in endurance runners: can jumping kinematic explain the post-activation potentiation phenomenon? Sports Biomech, 2016; 15: 103-115

Giovanelli N, Taboga P, Rejc E, Simunic B, Antonutto G, Lazzer S. Effects of an Uphill Marathon on Running Mechanics and Lower-Limb Muscle Fatigue. International Journal of Sports Physiology and Performance, 2016; 11: 522-529

Gollnick PD, Pihel K, Saltin B. Selective glycogen depletion pattern in human skeletal muscle fibres after exercise of varying intensity and at varying pedalling rates. J Physiol, 1974; 241: 45-57

Gołaś A, Maszczyk A, Zajac A, Mikolajec K, Stastny P . Optimizing Post Activation Potentiation for Explosive Activities in Competitive Sports. J Hum Kinet, 2016; 52 (1): 95-106

Hamada T, Sale DG, Macdougall JD. Postactivation potentiation in endurance-trained male athletes. Med Sci Sports Exerc, 2000; 32(3): 403-411

Healy R, Comyns TM. The application of postactivation potentiation methods to improve sprint speed. Strength and Conditioning Journal, 2017; 39(1): 1-9

Hodgson M, Docherty D, Robbins D. Post-activation potentiation: underlying physiology and implications for motor performance. Sports Med, 2005; 35(7): 585-595

Krustrup P, Soderlund K, Mohr M, Bangsbo J. Slow-Twitch Fiber Glycogen Depletion Elevates ModerateExercise Fast-Twitch Fiber Activity and O2 Uptake. Med Sci Sports Exercise, 2004; 36: 73-83

Latorre-Román PA, García-Pinillos F, Martínez-López EJ, Soto-Hermoso VM. Concurrent fatigue and postactivation potentiation during extended interval training in long-distance runners. Mot Rev Educ Física, 2014; 20: 423-430

Maszczyk A, Golas A, Czuba M, Krol H, Wilk M, Stastny P, Goodwin J, Kostrzewa M, Zajac A. EMG Analysis and Modelling of Flat Bench Press Using Artificial Neural Networks. SAJRPER. 2016; 38(1): 91-103.

Mettler JA, Griffin L. Postactivation potentiation and muscular endurance training. Muscle and Nerve, 2012; 45: 416-425

Meyer T, Gabriel HH, Kindermann W. Is determination of exercise intensities as percentages of VO2max or HRmax adequate? Med Sci Sports Exerc, 1999; 31: 1342-1345

Millet GY, Lepers R, Maffiuletti NA, Babault N, Martin V, Lattier G. Journal of Applied Physiology, 2002; 92: 486-492

Millet GY, Lepers R. Alterations of neuromuscular function after prolonged running, cycling and skiing exercises. Sports Medicine, 2004; 34: 105-116

Newell J, Higgins D, Madden N, Cruickshank J, Einbeck J, McMillan K, McDonald R. Software for calculating blood lactate endurance markers. J Sports Sci, 2007; 25: 1403-1409

Pueo B, Lipinska P, Jiménez-Olmedo JM, Zmijewski P, Hopkins WG. Accuracy of Jump-Mat Systems for Measuring Jump Height. Int J Sports Physiol Perform, 2017; 12(7): 959-963 
Raeder C, Wiewelhove T, Simola RÁ, Kellmann M, Meyer T, Pfeiffer M, Ferrauti A. Assessment of Fatigue and Recovery in Male and Female Athletes After 6 Days of Intensified Strength Training. J Strength Cond Res, 2016; 30(12): 3412-3427

Sams ML, Sato K, DeWeese BH, Sayers AL, Stone MH. Quantifying changes in squat jump height across a season of men's collegiate soccer. J Strength Cond Res, 2018; 32(8): 2324-2330

Seiler KS, Kjerland GO. Quantifying training intensity distribution in elite endurance athletes: is there evidence for an "optimal" distribution? Scandinavian Journal of Medicine E Science in Sports, 2006; 16: 4956

Tanner RK, Fuller KL, Ross MLR. Evaluation of three portable blood lactate analysers: Lactate Pro, Lactate Scout and Lactate Plus. European Journal of Applied Physiology, 2010; 109: 551-559

Tillin NA, Bishop D. Factors modulating post-activation potentiation and its effect on performance of subsequent explosive activities. Sports Medicine, 2009; 39: 147-166

Vuorimaa T, Virlander R, Kurkilahti P, Vasankari T, Häkkinen K. Acute changes in muscle activation and leg extension performance after different running exercises in elite long distance runners. Eur J Appl Physiol, 2006; 96: 282-291

Wilson GJ, Murphy AJ. The use of isometric tests of muscular function in athletic assessment. Sports Med, 1996; 22(1): 19-37

Wilson J, Duncan N, Marin P, Brown L, Loenneke J, Wilson S, Ugrinowitsch C. Meta-analysis of postactivation potentiation and power: effects of conditioning activity, volume, gender, rest periods, and training status. Journal of Strength and Conditioning Research, 2013; 27: 854-859

Winter EM, British Association of Sport and Exercise Sciences. Sport and exercise physiology testing guidelines: The British Association of Sport and Exercise Sciences guide. New York, NY: Routledge; 2006

\section{Corresponding author:}

\section{Marco Gervasi}

Affiliation: Department of Biomolecular Sciences, University of Urbino Carlo Bo, Urbino, Italy

Address: Via I Maggetti 26/2, 61029 Urbino (PU), Italy

Telephone number: +390722303413

Fax number: +390722303401

Email: marco.gervasi@uniurb.it

ORCID: 0000-0001-7112-1780 